\title{
A new influenza reassortant - a beast in the making
}

With generic consensus on the certainty of another impending influenza pandemic, concerns are mounting about its devastating global impact. Influenza pandemics of swine and avian origin have plagued mankind for centuries in the past. The current novel H1N1 influenza virus of swine origin was first isolated in mid-April 2009 and, by the end of the month information about its genomic sequence was known which projected it to be a novel reassortant. The virus spreads with ease and rapidly in the human population, and the resulting pandemic has already proved to be a significant and very costly cause of mortality and morbidity to humans.

This current reassortant $(\mathrm{H} 1 \mathrm{~N} 1 \mathrm{~A} /$ swine/ California/04/2009) is thought to be a fourth generation descendant of the 1918 pandemic H1N1 influenza A virus and appears to have originated in Mexico. As the virus spread across the globe with ease, it forced the WHO to raise the pandemic level alert to phase 5 on April 29 and further to phase 6 on June 11, 2009. This ongoing outbreak of novel influenza A H1N1 has not only caused more than 399,232 laboratory confirmed cases and over 6,000 deaths globally but has also exhibited transmission from person to person at higher transmissibility than of seasonal influenza viruses.

In India the first death of a 14-year-old girl on August 3, 2009 in the city of Pune raised fears of the pandemic in a country with high population density, low awareness and a poor health-care infrastructure. With these, the total number of laboratory-confirmed cases of the virus reported thus far in India has risen to 13,232. Unlike other affected countries, the victims in India have been mostly within the age group of 15-45 years. This is of concern since about half the Indian population is under the age of 25 and only $10 \%$ in the above 60 years age group. With seasonal flu however things are different, about 500,000 people die of seasonal flu worldwide every year, most of them senior citizens. As of October 23, 2009, the total death toll due to this pandemic has reached 431 . Of the total swine flu deaths reported in India till now, Maharashtra now accounts for 185 , while 112 people have lost their lives in Karnataka, 46 in Andhra Pradesh, 39 in Gujarat, 16 in Delhi, 12 in Kerala, 4 each in Tamil Nadu, Goa and Haryana, 3 each in Uttar Pradesh and Uttarakhand and
1 each in Chhattisgarh, Himachal Pradesh and Rajasthan. The city of Pune has seen the most deaths from the H1N1 flu pandemic in India. As we celebrate the Golden Jubilee of the Association of Microbiologists of India with their annual meeting in Pune, we dedicate this issue of the Indian Journal of Microbiology to the city of Pune and salute its communities for showing resilience and boldly facing the challenge of this deadly H1N1 virus.

We currently sit on the threat of a second more deadly wave of pandemic influenza and the World Health Organization predicts that some 2 billion people will get infected by the end of the current pandemic. With these huge numbers in hand adequate amounts of vaccine or anti-virals are unlikely to be available in a more severe pandemic, and the latter could become ineffective because of resistance. These factors have focused our attention on the use of non-pharmaceutical public health interventions to contain the infection, delay spread, and reduce the impact of pandemic disease. As microbiologists we recognize the need of the hour is more awareness of the disease, simple and inexpensive methods to curtail its spread. These are some of the lessons learnt.

First is to strengthen our monitoring systems, to give us better surveillance and epidemiology. Second is to improve the ability of state and local health authorities to implement public health and awareness programs, including non-pharmaceutical interventions. Let us not forget that animal surveillance is equally important to closely monitor the appearance of new respiratory pathogens. Production of any pandemic vaccine would take a minimum of 6 months after isolation of a novel virus, and antivirals may not be available where they are required most. Efforts to create a universal pool of resources and stockpiles of antivirals, antibiotics and vaccines for use in first affected countries may simply be plans that look good on paper. Preparedness and preventive approaches adopted by nation states are bound to get polarised between resource rich and resource challenged countries. In India, prevention will be our best defense. The principal focus of interventions against pandemic influenza spread will have to be at the national and community levels. Proper implementation of non-pharmaceutical public health interventions will therefore be vital in curtailing disease spread. 
Typically during a pandemic period, the focus shifts to delaying spread and reducing effects through populationbased measures. The patient acquires the infection from friends, family or close group members like in workplaces and public places via the mode of coughing, sneezing and unhygienic practices which result in quick transmission of this virus. It becomes important at this stage for us to break this transmission cycle of the virus by educating and implementing hygienic practices in our lifestyle and avoiding crowded gatherings where the virus gets a good opportunity for person-to-person propagation. If the pandemic is severe, social distancing measures such as school closures will be essential. Non-essential domestic travel to affected areas should be deferred. Hand and respiratory hygiene should be extensively promoted to the masses. Educating the masses on these lines will have a great impact on curtailing easy transmission of the virus.

As we come out with this special issue on the Swine Flu Pandemic, I can only emphasise more that a nationwide awareness program should be in place which educates communities of all age groups to take immediate steps to prevent transmission of this virus. These steps are easy and do not cost anything. They simply require a slight modification of lifestyle and hygiene practices. Maintain cleanliness in surroundings and keep oneself clean which means personal hygiene should be given great importance. While coughing and sneezing always cover your face so that there is no chance of transmission of any kind infection to people around us. People are advised to prevent close associations in public places, avoiding crowded place, avoiding usage of unhygienic public utilities and avoiding exposing oneself to infected person or infection prone areas. Let us educate our young to begin a "non-cooperation movement" against the virus to not cooperate with H1N1 and its likes, in their propagation from human to human.

Sunil K. Lal Guest Editor, Special Issue on the Swine Flu Pandemic International Centre for Genetic Engineering \& Biotechnology New Delhi, India sunillal@icgeb.res.in 